\title{
Analysis and application of two-current-source circuit as a signal conditioner for resistive sensors
}

\author{
Adam Idzkowski, Jerzy Gołębiowski, Wojciech Walendziuk*
}

\begin{abstract}
The article presents the analysis of metrological properties of a two-current-source supplied circuit. It includes such data as precise and simplified equations for two circuit output voltages in the function of relative resistance increments of sensors. Moreover, graphs showing nonlinearity coefficients of both output voltages for two resistance increments varying widely are presented. Graphs of transfer resistances, depending on relative increments of sensors resistance were also created. The article also contains a description of bridge-based circuit realization with the use of a computer and a data acquisition (DAQ) card. Laboratory measurement of the difference and sum of relative resistance increments of two resistance decade boxes were carried out indirectly with the use of the created measurement system. Measurement errors were calculated and included in the article, as well.
\end{abstract}

K e y w or d s: error analysis, measurement techniques, sensor systems

\section{Introduction}

Measurement methods exploit classic bridge circuits as transducers of resistance changes into voltage and use them for preliminary signal conditioning $[1,2]$. The accuracy of the bridge circuit depends on resistance precision placed in its branch and has significant influence on precision of the whole measurement chain [3]. Theoretic works concerning new possibilities of parallel measurement of two or more quantities (or their components) have appeared in literature since 2000 . The research is done with the use of classic or unconventional bridge-based measurement systems [4-6].

In [4] using those systems to build new measurement devices was also taken into consideration. The change of the way of supplying the bridge (using two direct current sources) was the novelty of this solution. Following works presented the idea of a two-current-supply automatically charge-balanced bridge [5]. According to the authors' knowledge, however, this research has not been continued.

Other solutions presented in literature are based on classic Wheatstone's bridge architecture. Unfortunately, they have separate sensors for each measured quantity, separate channels of classical unbalanced bridges and one common (current or voltage) power source. Output voltages are then measured sequentially, by switching from one bridge circuit to another. Continuous switching arms of a bridge and measuring the output voltage in each cycle is another way of measuring many physical quantities simultaneously. Anderson's loop, worked out for NASA purposes in the 1990s, is an alternative solution for classical unbalanced bridge $[7,8]$. Resistance sensors in such a system are connected serial to a power supply and a reference resistor. The measurement of resistance increments is done through subtraction voltages with the use of functional blocks equipped with measurement amplifiers.

Typically, resistive elements are combined into full Wheatstone bridge, for example in AMR sensors [9].

Because of the shortage of works concerning systems used for simultaneous measurement of many physical quantities [10] or impedance components [11], the authors of this paper decided to address the problem of constructing improved two-current-supply circuits with analogue voltage outputs. The research resulted in designing and building original prototypes of unbalanced bridges supplied by two identical or switchable DC current sources. It was assumed that, in specific circumstances, those circuits can be used as an alternative for commonly known and applied solutions for simultaneous measurement of two physical quantities. It is worth noting that the results of the research, which are presented in this article, have been patented [12].

\section{The analysis of a two-current supplied circuit}

A two-current-source bridge circuit described in [4] is only a theoretical solution. The circuit is supplied by two current sources galvanically isolated, and it does not have a permanent connection with the circuit ground. In order to test its metrological qualities, an attempt to physically realize the idea was taken. It was done through modifying the way of supply by introducing one, switchable current source [13].

This article suggests a different measurement system (Fig. 1). It does not have switchable current sources or

*Department of Electrical Engineering, Bialystok University of Technology, Wiejska 45D str., 15-351 Bialystok, Poland, a.idzkowski@pb.edu.pl, j.golebiowski@pb.edu.pl,w.walendziuk@pb.edu.pl 
any switching keys. Two sources supply the system permanently. Four resistance sensors $R_{1}, R_{2}, R_{3}, R_{4}$ are connected into a four-armed bridge. Two current sources, $J_{1}, J_{2}$ are joined up to the opposite nodes of the circuit. Two reference resistors $R_{r 1}, R_{r 2}$ (therefore: $2 \mathrm{~J}+2 \mathrm{R}$ ) are connected with the other nodes. It was assumed that current efficiency of the sources were identical and unchangeable in time $\left(J_{1}=J_{2}=\right.$ const $)$. This means that DC sources of high stability should be applied in this kind of systems. The currents of sources are channeled into A and $\mathrm{B}$ nodes.

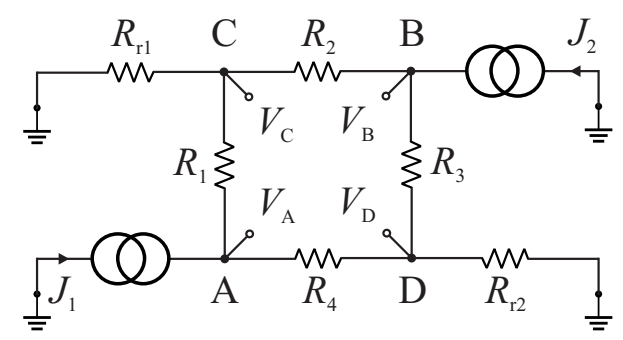

Fig. 1. Two - current - supply circuit $(2 J+2 R)$

The circuit described above measures the potential values for A, B, C and D nodes, or voltage between D-C and $\mathrm{A}-\mathrm{B}$ nodes. Below, a matrix equation describing dependences between electric values in this measurement system is presented. It shows that the measured potentials depend on resistance of particular arms of the system and current efficiency of both supplies

$$
\begin{gathered}
{\left[\begin{array}{cccc}
\frac{R_{1}+R_{4}}{R_{1} R_{4}} & 0 & -\frac{1}{R_{1}} & -\frac{1}{R_{4}} \\
0 & \frac{R_{2}+R_{3}}{R_{2} R_{3}} & -\frac{1}{R_{2}} & -\frac{1}{R_{3}} \\
-\frac{1}{R_{1}} & -\frac{1}{R_{2}} & \frac{R_{1} R_{2}+\left(R_{1}+R_{2}\right) R_{r 1}}{R_{1} R_{2} R_{r 1}} & 0 \\
-\frac{1}{R_{4}} & -\frac{1}{R_{3}} & 0 & \frac{R_{3} R_{4}+\left(R_{3}+R_{4}\right) R_{r 2}}{R_{3} R_{4} R_{r 2}}
\end{array}\right] \times} \\
\times\left[\begin{array}{lllll}
V_{A} & V_{B} & V_{C} & V_{D}
\end{array}\right]^{\top}=\left[\begin{array}{llll}
J_{1} & J_{2} & 0 & 0
\end{array}\right]^{\top}
\end{gathered}
$$

where $V_{A}, V_{B}, V_{C}, V_{D}$ are the potentials in $A, B, C$ and $D$ nodes.

Resistances in arms change under influence of measured quantities (eg deflection or temperature). It is presented in the following equation

$$
R_{i}=R_{i 0}\left(1+\varepsilon_{i}\right), \quad \text { for } \quad i=1,2,3,4,
$$

where $R_{i 0}$ is the initial (nominal) resistance, $\Delta R_{i}$ is the absolute resistance increment, $\varepsilon_{i}=\Delta R_{i} / R_{i 0}$ is the relative resistance increment of the $i$-th sensor.

As it was mentioned previously, equal values of current in both sources were assumed and also the values of reference resistors are equal the nominal resistance of the sensors

$$
\begin{gathered}
J=J_{1}=J_{2}, \\
R_{10}=R_{20}=R_{30}=R_{40}=R_{0}, \\
R_{r 1}=R_{r 2}=R_{r}=R_{0} .
\end{gathered}
$$

Solving equation (1) and substituting (2), (3a), (3b) and (3c) made the following formulas:

$$
\begin{gathered}
V_{A}=\frac{3 J R_{0}\left(6+\varepsilon_{1}+\varepsilon_{4}\right)}{12+4\left(\varepsilon_{1}+\varepsilon_{2}+\varepsilon_{3}+\varepsilon_{4}\right)+\left(\varepsilon_{1}+\varepsilon_{4}\right)\left(\varepsilon_{2}+\varepsilon_{3}\right)}, \\
V_{B}=\frac{3 J R_{0}\left(6+\varepsilon_{2}+\varepsilon_{3}\right)}{12+4\left(\varepsilon_{1}+\varepsilon_{2}+\varepsilon_{3}+\varepsilon_{4}\right)+\left(\varepsilon_{1}+\varepsilon_{4}\right)\left(\varepsilon_{2}+\varepsilon_{3}\right)}, \\
V_{C}=\frac{V_{A}\left(1+\varepsilon_{2}\right)+V_{B}\left(1+\varepsilon_{1}\right)}{2+\varepsilon_{1}+\varepsilon_{2}+\left(1+\varepsilon_{1}\right)\left(1+\varepsilon_{2}\right)}, \\
V_{D}=\frac{V_{A}\left(1+\varepsilon_{3}\right)+V_{B}\left(1+\varepsilon_{4}\right)}{2+\varepsilon_{3}+\varepsilon_{4}+\left(1+\varepsilon_{3}\right)\left(1+\varepsilon_{4}\right)} .
\end{gathered}
$$

The following step was to calculate output voltages of the circuit with one pair of sensors which means the calculation was done for two increments $\varepsilon_{1}, \varepsilon_{2} \neq 0$, and two other $\varepsilon_{3}=\varepsilon_{4}=0$

$$
V_{D}=\frac{V_{A}\left(1+\varepsilon_{3}\right)+V_{B}\left(1+\varepsilon_{4}\right)}{2+\varepsilon_{3}+\varepsilon_{4}+\left(1+\varepsilon_{3}\right)\left(1+\varepsilon_{4}\right)}
$$

and

$$
U_{D C}=V_{D}-V_{C}=J R_{0} \frac{K(\varepsilon)}{H(\varepsilon)}
$$

where:

$$
\begin{aligned}
K(\varepsilon) & =6\left(\varepsilon_{1}+\varepsilon_{2}\right)+10 \varepsilon_{1} \varepsilon_{2}+\varepsilon_{1}\left(2 \varepsilon_{1}+\varepsilon_{1} \varepsilon_{2}\right) \\
& +\varepsilon_{2}\left(2 \varepsilon_{2}+\varepsilon_{1} \varepsilon_{2}\right) \\
H(\varepsilon) & =36+36\left(\varepsilon_{1}+\varepsilon_{2}\right)+15 \varepsilon_{1} \varepsilon_{2}+8\left(\varepsilon_{1}+\varepsilon_{2}\right)^{2} \\
& +6\left(\varepsilon_{1}+\varepsilon_{2}\right) \varepsilon_{1} \varepsilon_{2}+\varepsilon_{1}^{2} \varepsilon_{2}^{2} .
\end{aligned}
$$

The above equations show that for high relative increments $\varepsilon_{i} \gg 0$ the voltages $U_{A B}$ and $U_{D C}$ are non-linear functions of relative increments $\varepsilon_{i}$. Whereas, for sensors with low resistance increment $\varepsilon_{1}, \varepsilon_{2}$ (when the following conditions occur: $4\left|\varepsilon_{1}+\varepsilon_{2}\right| \ll 12,36\left|\varepsilon_{1}+\varepsilon_{2}\right| \ll 36$ (it results that $\left|\varepsilon_{1}+\varepsilon_{2}\right| \ll 1$ ), $\varepsilon_{1}^{2} \approx 0, \varepsilon_{2}^{2} \approx 0$ and $\varepsilon_{1} \varepsilon_{2} \approx 0$ ), both functions become linear. Making those assumptions, equations (8) and (9) can be simplified so that linear forms of the functions (10) and (11) are obtained

$$
\begin{aligned}
& U_{A B u} \cong V_{A}-V_{B}=\frac{J R_{0}}{4}\left(\varepsilon_{1}-\varepsilon_{2}\right), \\
& U_{D C u} \cong V_{D}-V_{C}=\frac{J R_{0}}{6}\left(\varepsilon_{1}+\varepsilon_{2}\right) .
\end{aligned}
$$

The analysis of the equations (10) and (11) proves that the system can work with one pair of resistance sensors. As a result, measuring two increments at the same time, as well as the sum and difference of resistances is possible. This conditions and formulas can be useful in applications with strain gauges, especially when a resistance sensor is differential [14]. 


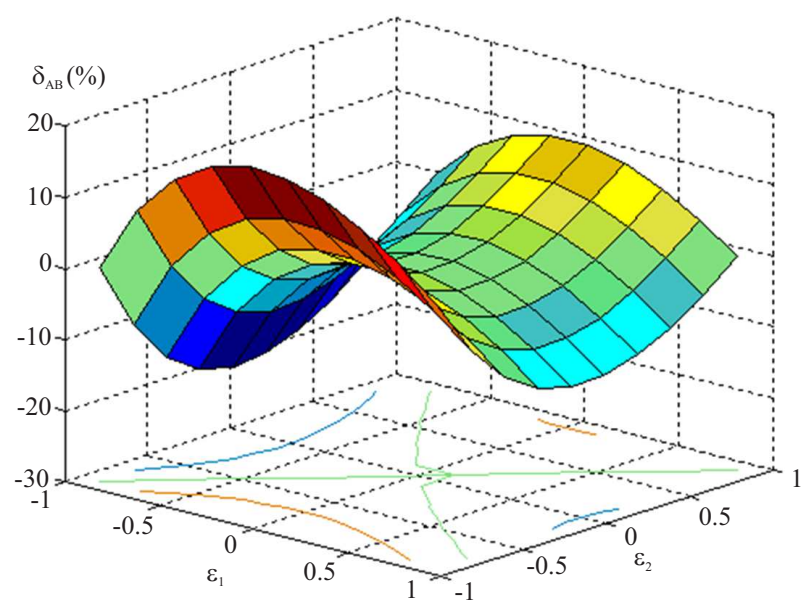

Fig. 2. $\delta_{A B}$ nonlinearity coefficient in function of $\varepsilon_{1}$ and $\varepsilon_{2}$

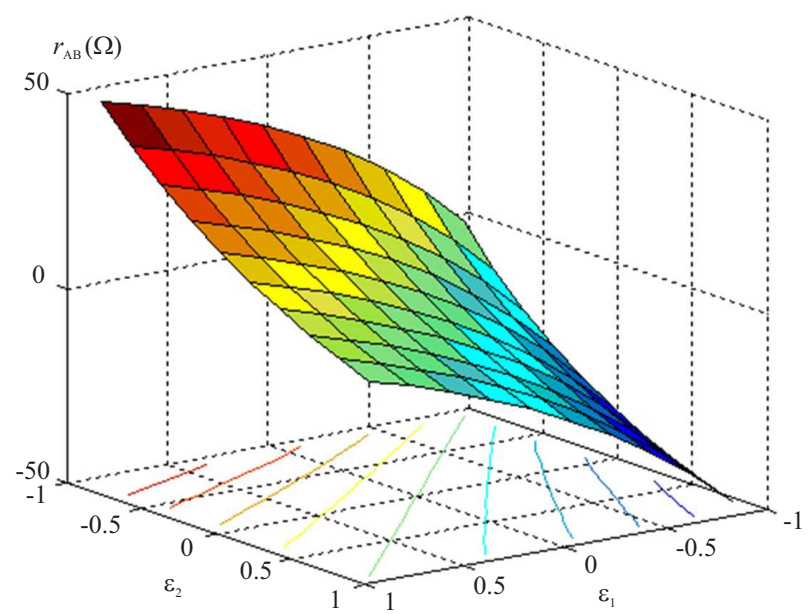

Fig. 4. Transfer resistance $r_{A B}$ of transducer in relation to $\varepsilon_{1}$ and $\varepsilon_{2}\left(\right.$ when $\left.R_{r}=R_{0}=100 \Omega\right)$

Differences between linearized equations (10), (11) and the real ones $(8),(9)$ are described with the use of relative non-linearity coefficients of $\delta_{A B}$ and $\delta_{D C}$. These are absolute non-linearity errors [15] related to full measurement ranges of both output voltages

$$
\begin{aligned}
& \delta_{A B}=\frac{U_{A B u}-U_{A B}}{U_{A B \max }-U_{A B \min }} \cdot 100 \%, \\
& \delta_{D C}=\frac{U_{D C u}-U_{D C}}{U_{D C \max }-U_{D C \min }} \cdot 100 \%,
\end{aligned}
$$

where $U_{A B \text { max }}-U_{A B \text { min }}=\Delta_{A B}, U_{D C \text { max }}-U_{D C \text { min }}=$ $\Delta_{D C}$ are full ranges of output voltages of the system calculated for minimum $(-0.9)$ and maximum $(0.9)$ value of variable $\varepsilon_{2}$ where $\varepsilon_{1}$ is given

$$
\begin{aligned}
& \Delta_{A B}=U_{A B}\left(\varepsilon_{1}, \varepsilon_{2}=0.9\right)-U_{A B}\left(\varepsilon_{1}, \varepsilon_{2}=-0.9\right), \\
& \Delta_{D C}=U_{D C}\left(\varepsilon_{1}, \varepsilon_{2}=0.9\right)-U_{D C}\left(\varepsilon_{1}, \varepsilon_{2}=-0.9\right) .
\end{aligned}
$$

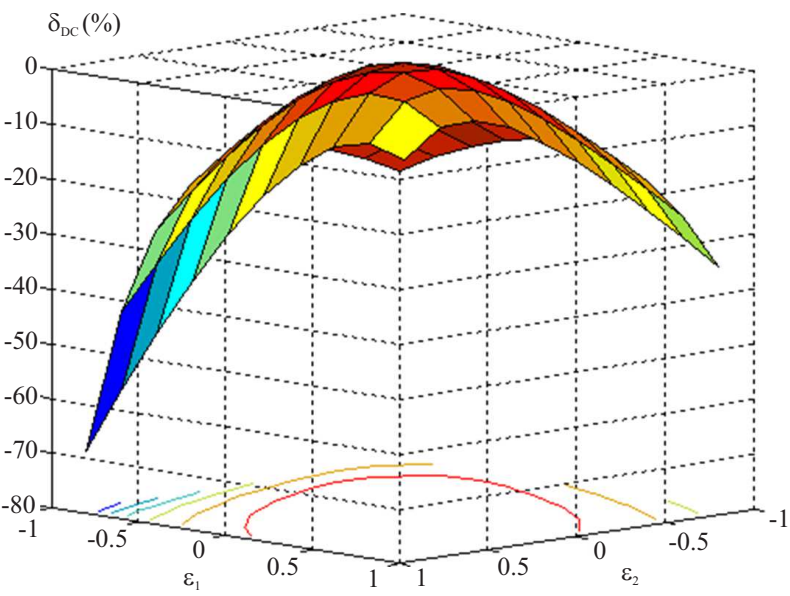

Fig. 3. $\delta_{D C}$ nonlinearity coefficient in function of $\varepsilon_{1}$ and $\varepsilon_{2}$

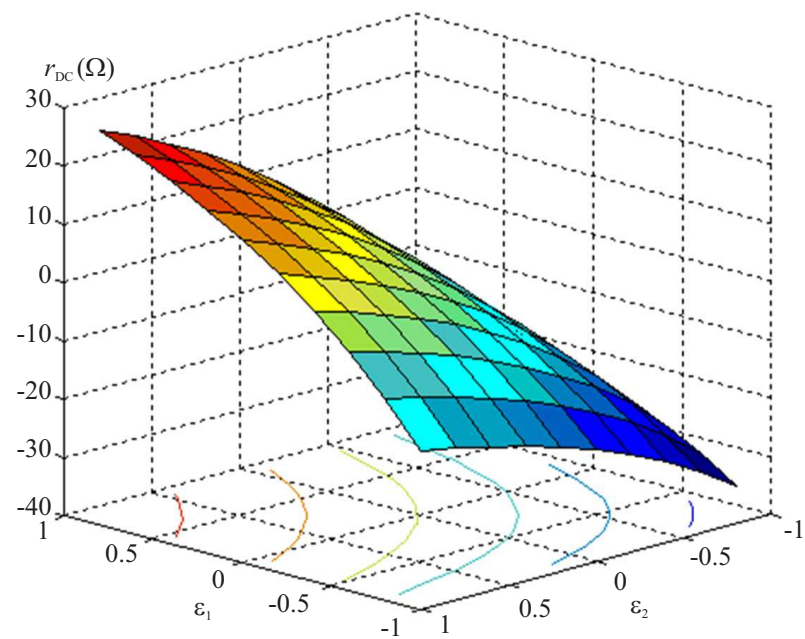

Fig. 5. Transfer resistance $r_{D C}$ of transducer in relation to $\varepsilon_{1}$ and $\varepsilon_{2}\left(\right.$ when $\left.R_{r}=R_{0}=100 \Omega\right)$

The graphs of relative non-linearity coefficients (12), (13) are presented in Figs. 2-5 (for $J=100 \mathrm{~mA}$ and $R_{0}=100 \Omega$ ).

Comparing Fig. 2 and Fig. 3 proves that for negative values of $\varepsilon_{1}$ parameter, the range of changes of the $\delta_{A B}$ non-linearity coefficient takes higher values (from about $-20 \%$ to about $+20 \%$ ) than at $\varepsilon_{1}>0$ (from about $-10 \%$ to about $+20 \%)$. It can be observed that for $\varepsilon_{1}<0\left|\delta_{D C}\right|$ it takes a slightly higher value (about $70 \%$ ) than for $\varepsilon_{1}>0$ (about $35 \%$ ).

Relative increments of resistance in strain gauge sensors are significantly lower than the limiting values \pm 0.9 assumed above. For this reason, some additional computations for $\left|\varepsilon_{1}\right|<0.1$ and $\left|\varepsilon_{2}\right|<0.1$ were carried out. Modules of non-linearity coefficients $\left|\delta_{A B}\right|$ and $\left|\delta_{D C}\right|$ within this range are not higher than $0.2 \%$ and $0.5 \%$, respectively. 


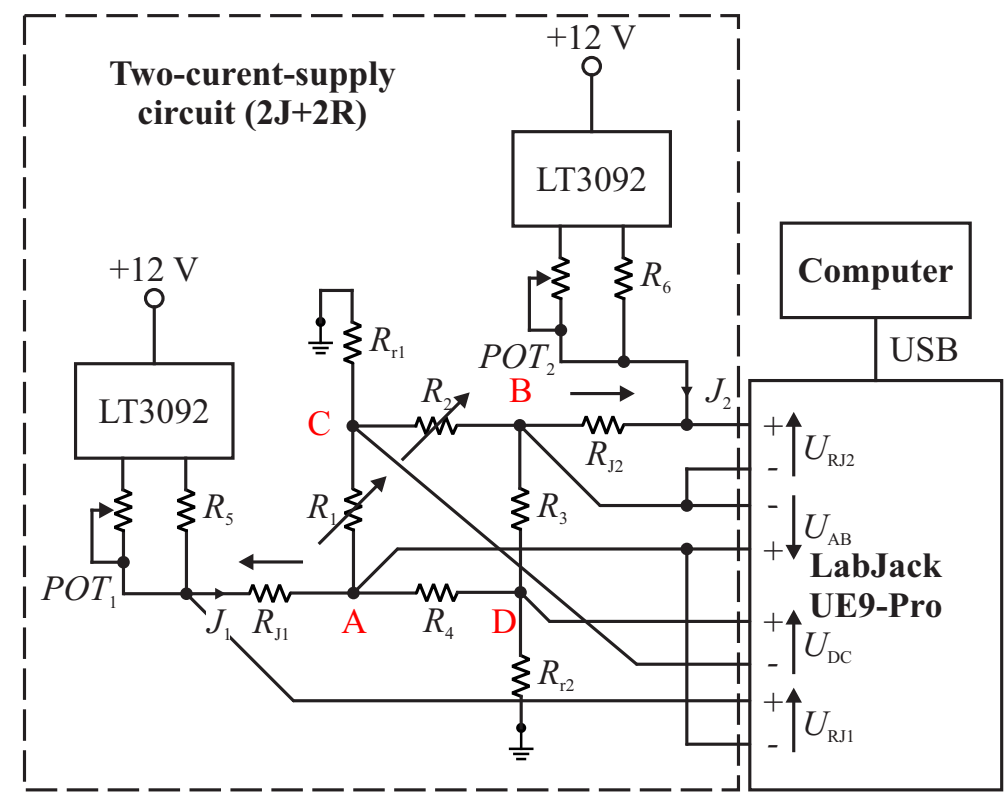

Fig. 6. Measurement system consisting of a two - current - supply circuit $(2 j+2 R)$, a LabJack measurement module (UE9-Pro) and a $\mathrm{PC}$

Moreover, $r_{A B}$ and $r_{D C}$ transfer resistances were defined as circuit output voltage to supply current $J$

$$
\begin{aligned}
& r_{A B}=\frac{U_{A B}}{J}, \\
& r_{D C}=\frac{U_{D C}}{J}
\end{aligned}
$$

where (8) and (9) were applied. Figures 4 and 5 present $r_{A B}$ and $r_{D C}$ graphs in the function of $\varepsilon_{1}, \varepsilon_{2}$ resistance relative increments.

Comparing Figs. 4 and 5 shows that $r_{A B}$ decreases and $r_{D C}$ grows in a function of increasing $\varepsilon_{2}$ value (assuming that $\varepsilon_{1}$ value is constant). Moreover, the range of $r_{A B}$ function changes is larger than for $r_{D C}$ function.

\section{Two-current-supply circuit realization}

A two-current-supply circuit was designed and built. LT3092 systems were used as current sources (Fig. 6). They have great stability and can be regulated within 0.5-200 mA range, and additionally - which is an advantage - do not require applying external capacitors. Their latency time is short (about $20 \mu \mathrm{s}$ ). The accuracy of the sources currents is $1 \%$ and the temperature drift $-0.3 \%$ within the range of $0-100{ }^{\circ} \mathrm{C}$ (at the working current of $1 \mathrm{~mA}$ ). The sources currents depend on the values adjusted on potentiometers (POT1 and POT2) and on resistance values $\left(R_{5}\right.$ and $\left.R_{6}\right)$. The value of the sources current, which was precisely set, equalled $J=100 \mathrm{~mA}$ and was controlled during experiments through measuring voltage drop $\left(U_{R J 1}\right.$ and $\left.U_{R J 2}\right)$ on resistors $\left(R_{J 1}\right.$ and $\left.R_{J 2}\right)$. Reference resistors of the $R_{r 1}=R_{r 2}=100 \Omega$ value are joined with $\mathrm{C}$ and $\mathrm{D}$ points and their free endings - to the ground of the system. High precision and stability of resistance values in the function of temperature should be their features.

In order to conduct the research, a measurement system consisting of two-current-supply circuit $(2 \mathrm{~J}+2 \mathrm{R})$, a UE9-Pro LabJack data acquisition module and a PC were used. The LabJack module was equipped with unipolar inputs of the range of $0-5 \mathrm{~V}$. This causes voltage resolution of measurement equal $4.8 \mu \mathrm{V}$ at the Analog to Digital Converter set resolution of 20 bits (resolution may change from 16 to 24 bits).

Two resistance decades (MDR-93/2-4a) play the role of variable resistors $R_{1}$ and $R_{2}$ in the circuit. They were used to set chosen values of resistance increase of sensors. A computer program created in LabVIEW environment was used to acquire, visualize, and store data in a file [16].

\section{Laboratory research}

The research was aimed at indirect calculating the values of $\varepsilon_{R}=\varepsilon_{1}-\varepsilon_{2}$ and $\varepsilon_{S}=\varepsilon_{1}+\varepsilon_{2}$ of the increments of $R_{1}$ and $R_{2}$ decade resistors relative resistance. For this reason, different combinations of $\varepsilon_{1}$ and $\varepsilon_{2}$ relative increments were placed on decades, assuming that $\mid \varepsilon_{1}+$ $\varepsilon_{2} \mid \ll 1$ (condition in accordance with assumptions of asymptotic formulas validity (10), (11)).

The change of decade resistance $R_{1}$ with a step of $\Delta R_{1}=+2.00 \Omega$ (which is a value according to $R_{0}=$ $100 \Omega$ that equals $\left.\varepsilon_{1}=+0.02\right)$ and $R_{2}$ with a step of $\Delta R_{2}=-1.00 \Omega$ (which is $\varepsilon_{2}=-0.01$ ) resistance can be given as examples of this case. Then, the difference of relative increments equals $\varepsilon_{R}=\varepsilon_{1}-\varepsilon_{2}=0.03$ and the sum $-\varepsilon_{S}=\varepsilon_{1}+\varepsilon_{2}=0.01$. After each decade resistance being set in this way, measurement of voltage in both diagonals of the bridge $\left(U_{A B}\right.$ and $\left.U_{D C}\right)$ was conducted. 
Average values $\left(\bar{U}_{A B}\right.$ and $\left.\bar{U}_{D C}\right)$ and standard deviations $\sigma_{U A B}, \sigma_{U D C}$ (Tab. 1) were calculated on the basis of simultaneously recorded instantaneous voltage $\left(U_{A B}\right.$ and $\left.U_{D C}\right)$ in series of 1000 samples. It can be observed in Tab. 1 that the $\sigma_{U D C} \gg \sigma_{U A B}$ inequality occurs for a given variant of decades setting. This proves that voltage functions of $U_{D C}$ are greater than those of $U_{A B}$.

Table 1. Measurement results of average voltage values at the diagonals of the circuit for different combinations of relative resistance increments (1000 samples acquired every $0.1 \mathrm{~s}$ ).

\begin{tabular}{lcccccc}
\hline & \multicolumn{3}{c}{ Set values } & \multicolumn{4}{c}{ Measured values } \\
\cline { 2 - 7 } No. & $\varepsilon_{1}$ & $\varepsilon_{2}$ & $\bar{U}_{A B}$ & $\sigma_{U A B}$ & $\bar{U}_{D C}$ & $\begin{array}{c}\sigma_{U D C} \\
(\mathrm{mV})\end{array}$ \\
& - & - & $(\mathrm{mV})$ & $(\mathrm{mV})$ & $(\mathrm{mV})$ \\
\hline 1 & 0.00 & 0.00 & 0.0019 & 0.0005 & 1.1138 & 0.6392 \\
2 & 0.00 & -0.01 & -18.289 & 0.0028 & 27.163 & 0.4166 \\
3 & 0.00 & -0.02 & -34.899 & 0.0340 & 52.461 & 0.5280 \\
4 & 0.01 & -0.01 & -1.772 & 0.0118 & 51.611 & 0.4873 \\
5 & 0.01 & -0.02 & -18.288 & 0.0036 & 77.415 & 0.3463 \\
6 & 0.02 & -0.01 & 15.300 & 0.0021 & 75.979 & 0.3977 \\
7 & 0.02 & -0.02 & -1.797 & 0.0031 & 94.462 & 0.3875 \\
\hline
\end{tabular}

Then, equations (10) and (11) were transformed to the following forms

$$
\begin{aligned}
& \bar{\varepsilon}_{R W}=4 \frac{\bar{U}_{A B}}{J R_{0}}, \\
& \bar{\varepsilon}_{S W}=6 \frac{\bar{U}_{D C}}{J R_{0}} .
\end{aligned}
$$

Differences and sums of two relative resistance increments for average voltage values $\bar{U}_{A B}, \bar{U}_{D C}$ were calculated according to (18) and (19). Further, relative differences $e_{R}$ and $e_{S}$ between the calculated (average) $\bar{\varepsilon}_{R W}$ and $\bar{\varepsilon}_{S W}$ values, and values $\varepsilon_{R}$ and $\varepsilon_{S}$ set on the decades were defined.

$$
\begin{aligned}
& \left|e_{R}\right|=\frac{\left|\varepsilon_{R}\right|-\left|\bar{\varepsilon}_{R W}\right|}{\left|\varepsilon_{R}\right|} \cdot 100 \%, \\
& \left|e_{S}\right|=\frac{\left|\varepsilon_{S}\right|-\left|\bar{\varepsilon}_{S W}\right|}{\left|\varepsilon_{S}\right|} \cdot 100 \% .
\end{aligned}
$$

Table 2 contains differences and sums of $\varepsilon_{1}$ and $\varepsilon_{2}$ relative increments. Moreover, values of $e_{R}$ and $e_{S}$ relative differences are given there. As it can be observed, the differences are not greater than $9.7 \%$ within the tested area $\varepsilon_{1}-\varepsilon_{2} \ll 0.04$.

\section{Conclusions}

The presented analysis of output voltage functions shows that, for $\left|\varepsilon_{1}+\varepsilon_{2}\right| \ll 1$ condition, simplified equations (10) and (11) can be applied to calculate $U_{A B}$ and $U_{D C}$ voltages. For changes of both parameters within $\left|\varepsilon_{1}\right| \leq 0.9$ and $\left|\varepsilon_{2}\right| \leq 0.9$, the nonlinearity coefficient module $\left|\delta_{A B}\right|$ increments up to $20 \%$ in extreme cases (Fig. 2), and $\left|\delta_{D C}\right|$ coefficient to $70 \%$ (Fig. 3). If the range of both parameters is shrunk to $\left|\varepsilon_{1}\right|<0.1$ and $\left|\varepsilon_{2}\right|<0.1$, the modules of coefficients $\left|\delta_{A B}\right|$ and $\left|\delta_{D C}\right|$ are not greater than $0.2 \%$ and $0.5 \%$ respectively. In the case of strain gauges, the $\varepsilon_{1}$ and $\varepsilon_{2}$ values change within the range of -0.02 to 0.02 . The values of both nonlinearity values are then placed within the range of $\pm 0.2 \%$. For this reason, equations (10) and (11) are precise enough.

The constructed bridge circuit (Figs. 6, 7) enabled, indirectly, laboratory measurement of $\varepsilon_{R}=\varepsilon_{1}-\varepsilon_{2}$ and $\varepsilon_{S}=\varepsilon_{1}+\varepsilon_{2}$ of resistance relative increments of $R_{1}$ and $R_{2}$ decade resistors. The measurements show that relative differences of sums and differences of $\bar{\varepsilon}_{R W}$ and $\bar{\varepsilon}_{S W}$ increments are not greater than $9.7 \%$ (Tab. 2). This proves that the constructed circuit (Figs. 6, 7) works (with acceptable error) according to created equations (10) and (11).

Another work will contain comparison of metrological properties of the bridge circuit with other circuits used for simultaneous measurement of two physical quantities.

\section{Acknowledgements}

The paper was prepared at Bialystok University of Technology within a framework of the S/WE/1/2013

\begin{tabular}{|c|c|c|c|c|c|c|c|c|}
\hline \multirow{3}{*}{ No. } & \multicolumn{4}{|c|}{ Set values } & \multicolumn{2}{|c|}{$\begin{array}{l}\text { Calculated from } \\
(18) \text { and (19) }\end{array}$} & \multicolumn{2}{|c|}{$\begin{array}{l}\text { Relative differences } \\
(20) \text { and }(21)\end{array}$} \\
\hline & $\varepsilon_{1}$ & $\varepsilon_{2}$ & $e_{R}$ & $e_{S}$ & $\bar{\varepsilon}_{R W}$ & $\bar{\varepsilon}_{S W}$ & $\left|e_{R}\right|$ & $\left|e_{S}\right|$ \\
\hline & - & - & - & - & - & - & $\%$ & $\%$ \\
\hline 1 & 0.00 & 0.00 & 0.00 & 0.00 & 0.00045 & 0.00000 & - & - \\
\hline 2 & 0.00 & -0.01 & 0.01 & -0.01 & 0.01086 & -0.01095 & 8.65 & 9.50 \\
\hline 3 & 0.00 & -0.02 & 0.02 & -0.02 & 0.02098 & -0.02094 & 4.92 & 4.70 \\
\hline 4 & 0.01 & -0.01 & 0.02 & 0.00 & 0.02064 & -0.00106 & 3.22 & - \\
\hline 5 & 0.01 & -0.02 & 0.03 & -0.01 & 0.03097 & -0.01097 & 3.22 & 9.70 \\
\hline 6 & 0.02 & -0.01 & 0.03 & 0.01 & 0.03039 & 0.00918 & 1.31 & 8.20 \\
\hline 7 & 0.02 & -0.02 & 0.04 & 0.00 & 0.03778 & -0.00108 & 5.54 & - \\
\hline
\end{tabular}
project.

Table 2. The values of relative resistance increments set into decades, values calculated by measurement equations, and received relative differences 


\section{REFERENCES}

[1] P. Sydenham and R. Thorn, Handbook of Measuring Systems Design, Chapter 127, John Wiley \& Sons, Ltd., 2005.

[2] N. A. Gilda, S. Nag, S. Patil, M. S. Baghini, D. K. Sharma and V. R. Rao, "Current Excitation Method for Delta R Measurement Piezo-Resistive Sensors With a 0.3-ppm Resolution", IEEE Transactions on Instrumentation and Measurement, vol. 61, no. 3, 2012, pp. 767-774, doi: 10.1109/TIM.2011.2172118.

[3] Z. L. Warsza and A. Idzkowski, "Errors and Uncertainties of Imbalanced Bridge-Circuits as Primary Converters for RTD sensors", Advanced Mechatronics Solutions, vol. 393 of the series Advances Intelligent Systems and Computing, Springer, 2015, pp. 397-410, doi: 10.1007/978-3-319-23923-1_60.

[4] Z. L. Warsza, "Two Parameter (2D) Measurements Double-Current Supply Four-Terminal Resistance Circuits", Metrology and Measurement Systems, vol. 13, no.1, 2006, pp. 49-65.

[5] J. Jakubiec, "Measuring Transducers based on Charge Balanced Resistance Bridge", Measurement Automation and Monitoring (PAK), 54 (5), 2008, pp. 280-285.

[6] C. Pedersen, S. T. Jespersen, J. P. Krog, C. Christensen and E. V. Thomsen, "Combined Differential and Static Pressure Sensor based on a Double-Bridged Structure", IEEE Sensors Journal 5 (3), 2005, pp. 446-454, doi: 10.1109/JSEN.2005.845199.

[7] K. F. Anderson, "Looking under the (Wheatstone) bridge", Sensors 18 (6), 2001.

[8] E. Jafer and C. S. Ibala, "Design and Development of MultiNode based Wireless System for Efficient Measuring of Resistive and Capacitive Sensors", Sensors and Actuators A: Physical, vol. 189, 2013, pp. 276-287, doi: 10.1016/j.sna.2012.09.023.

[9] V. Markevicius, D. Navikas, M. Zilys, D. Andriukaitis, A. Valinevicius and M. Cepenas, "Dynamic Vehicle Detection via the Use of Magnetic Field Sensors", Sensors, vol. 16, no.1, 2016, A.N.78, doi: 0.3390/s16010078.

[10] A. Reig, C. Demarcellis and M. D. Cubells-beltran, "Current-Based Measurement Technique for High Sensitivity Detection of Resistive Bridges with External Balancing Through Control Voltages", IEEE Sensors Journal, vol. 12, no.2, 2017, pp. 404-411, doi: 10.1109/JSEN.2016.2627640.

[11] A. Cichy, "Methods of Synthesis of Quasi-Balanced Circuits for Measuring of Impedance Components", Elektronika ir Elektrotechnika (Electronics and Electrical Engineering), vol. 22, no.2, 2016, pp. 38-42, doi: 10.5755/j01.eie.22.2.6463.

[12] W. Walendziuk and A. Idzkowski, "Double Current System for Sequential Measurement of Four Differences or Sums of Resistance Increments", Patent Number: PL221900(B1), 2016.

[13] A. Idzkowski, W. Walendziuk and Z. L. Warsza, "Unconventional Double-Current Circuit for Deflection and Temperature Simultaneous Measurement", Elektronika ir Elektrotechnika (Electronics and Electrical Engineering), vol. 21, no.1, 2015, pp. 23-27, doi: 10.5755/j01.eee.21.1.6834.

[14] GS. Zhao, HF. Pei and HC. Liang, "Measurement of Additional Strains Shaft Lining Using Differential Resistance Sensing Tech- nology", International Journal of Distributed Sensor Networks, A.N.153834, 2013, doi: 10.1155/2013/153834.

[15] W. Bolton, Instrumentation and Control Systems, Chapter 1, Elsevier Science \& Technology, 2015.

16] M. Penhaker, M. Cerny and M. Darebnikova, "Sensor Network for Measurement and Analysis on Medical Devices Quality Control" JJ. Yonazi et al (Eds.): ICeND 2011 CCIS 171, Springer-Verlag Berlin Heidelberg, 2011, pp. 182-196, doi: 10.1007/978-3-642-22729-5_16.

Received 3 November 2016

Adam Idzkowski (Engr Dr) graduated at Bialystok University of Technology. He received MSc in Electronics and Telecommunications 2001, PhD in Electrical Engineering 2013. Currently, he works as an assistant professor at Bialystok University of Technology. He is the author or coauthor of over 60 papers in the field of metrology, measurement systems and biomedical engineering, and a co-inventor of 2 national patents.

Jerzy Gołębiowski (Engr Prof) was born in Gliwice, Upper Silesia, Poland on 15 November 1953. He received his MSc degree in automatic control from the Silesian Technical University, Poland in 1977. He received his PhD degree from the Technical University of Szczecin, Western Pomerania, Poland in 1980, graduating with honors in electrical engineering. He also received the DSc degree in the field theory from the Silesian Technical University, in 1990. In 2011 he received the title of Professor from the President of Poland, after nomination from the Central Board for Scientific Degrees. He is the Head of the Chair for Theoretical Electrotechnics and Measurements, Technical University of Bialystok, Podlasie, Poland. His main research interests are in the analysis and synthesis of electromagnetic and thermal fields in electrical devices. Professor Golebiowski published ca 100 papers in regular scientific periodicals and four books. On invitation of foreign scientific centers, he regularly visits France, Israel and Canada.

Wojciech Walendziuk (Engr Dr) received his MSc degree in Electrical Engineering - Automation and Metrology in 1998, and $\mathrm{PhD}$ degree in Electrical Engineering in 2007, all from the Bialystok University of Technology, Bialystok. He is currently an assistant professor of Bialystok University of Technology, Faculty of Electrical Engineering, Department of Teoretical Electrotechnics and Metrology. He is a co-inventor of 2 national patents and the author of over 100 publications in the field of parallel processing, numerical methods, metrology, measurement of non-electrical quantities with the use of electrical methods. His main research interests include also signal analysis, signal conditioners and measurement systems application in medicine and Unmanned Aerial Vehicles. 